\title{
Changes in selected electrolytes in adult intensive care patients at the University Teaching Hospital, Lusaka, Zambia
}

\author{
Sheyo $\mathbf{N}^{1}$, Vwalika $\mathbf{B}^{2}$, and Kinnear $\mathbf{J}^{3}$ \\ 1. University of Zambia, School of Medicine, Department of Anaesthesia, P.O. Box 50110, Lusaka, Zambia and University \\ Teaching Hospital, Department of Anaesthesia, P/B RW1X, Lusaka, Zambia \\ 2. University of Zambia, School of Medicine, Department of Obstetrics and Gynaecology, P.O. Box 50110, Lusaka, Zambia \\ 3. Anglia Ruskin University, Head of Anglia Ruskin School of Medicine, Michael Salmon Building, Bishop Hall Lane, \\ Chelmsford | CM1 1SQ
}

Address of Correspondence:

Dr Ninza Sheyo; University Teaching Hospital, Department of Anaesthesia, P/B RW1X, Lusaka, Zambia.

Phone: +26096 744 0748; E-mail: ninza.sheyo@gmail.com

\begin{abstract}
Background: The importance of regulating electrolyte levels is well recognized in most Intensive Care Units (ICU). Various institutions across the globe have found varying figures on the extent and causes of electrolytes derangements in ICUs. The extent of electrolyte and fluid imbalance in patients being admitted and already admitted to the Main ICU (MICU) at the University Teaching Hospital (UTH) Lusaka Zambia is unknown.

This study aimed to explore the 24hour changes in sodium and potassium in adult MICU patients at the UTH, Lusaka, Zambia.
\end{abstract}

Methods: This was a prospective cohort study of the patients admitted to the MICU at UTH, Lusaka, Zambia. Data was collected over a fourmonth period (August to November) in 2017. Only consenting patients 18 years and above admitted for at least 24 hours in the unit were enrolled. On admission routine baseline investigations were obtained from every patient which included a full blood count and renal function tests. The second set of investigations was collected 24 hours post MICU admission. The blood samples were obtained from a peripheral vein in heparinized bottles for renal function tests. Serum electrolyte analysis was done was at UTH. Normal serum concentrations of sodium and potassium were considered as $135-145 \mathrm{mmol} / 1$ and $3.5-4.5 \mathrm{mmol} / \mathrm{L}$, respectively. Comparisons between means were done with the Wilcoxon signed rank test. Logistic regression analysis was used to investigate the relationship between dependent and independent variables. A $p$ - value $<0.05$ was considered statistically significant. Statistical analysis was performed with STATA 13 SE.
Results: A total number of 100 patients were included in this study with a mean age of 36.8 years $(\mathrm{SD}=12.1)$. The mean value of sodium level was $136.7(\mathrm{SD}=8.9) \mathrm{mmol} / \mathrm{L}$ and 139.0 $(\mathrm{SD}=11.6) \mathrm{mmol} / \mathrm{L}$, on admission and 24 hours post admission respectively. This difference in serum sodium level was shown to be statistically significant with a P-value $=0.0051$. Hypernatremia was shown to be associated with an increased risk of death $(\mathrm{p}=0.021)$ in the Unit with an odds ratio of 4.0 at $95 \%$ confidence interval of 1.3 to 13.8 . Hyponatremia was the most prevalent electrolyte imbalance but was neither shown to be associated with mortality $(\mathrm{P}$-value $=0.18)$ nor prolonged ICU stay (0.56) at 24 hours post admission. The mean value of potassium level was $4.2(\mathrm{SD}=1.1)$ $\mathrm{mmol} / \mathrm{L}$ and $4.3(\mathrm{SD}=1.1)$, on admission and 24 hours post admission respectively. This difference was not statistically significant $(\mathrm{P}$-value $=0.57$ ).

Conclusion: There was astatistically significant change in serum sodium levels after 24 hours post admission but there was no statistically significant change in potassium. Hyponatremia was the most prevalent abnormality whilst hypernatremia had astatistically significant association with mortality. Therefore, electrolyte imbalances can occur as early as the first day of admission in ICU with fatal complications. Correcting electrolyte imbalances in MICU patients is an urgent necessity and should not be delayed.

Keywords: Hypernatremia, Hyponatremia, Hypokalaemia, Hyperkalaemia, Electrolytes 


\section{INTRODUCTION}

Fluid and electrolyte balance are among the key physiologic processes in maintenance of body homeostasis, and plays very important roles in cellular function, myocardial function, neurological function, enzymatic function, tissue perfusion, oxygen delivery and acid base balancel . The Main Intensive Care Unit (MICU) at the University Teaching Hospital (UTH) is the largest ICU in the Republic of Zambia with a bed capacity of ten. Because of the limited bed capacity coupled with a high demand for critical care services, there is long waiting list for admission to the unit. Due to the high demand of critical cares services, patients need to be optimally treated for their lifethreatening conditions and transferred out of the MICU as soon as possible. This involves timely diagnosis and treatment of electrolyte disturbances that the patientsare admitted with or acquires during the stay in MICU.

Various institutions and hospitals across the globe have found varying figures on the extent and cause of electrolytes derangements in Intensive Care Units1,11,12,13.However, at UTH, there is no data on electrolyte changes in patients admitted to MICU with deranged electrolytes or those who acquire iatrogenic electrolyte disturbances during their Intensive Care Unit stay.This is because studies of this nature have not yet been done in ICUs across Zambia. The situation has remained so despite several studies regionally and internationally that have shown that electrolyte and acid base disturbances are common in ICU.

This study was aimed to ascertain changes in selected electrolytes in patients admitted to the MICU at UTH Lusaka, Zambia.

\section{METHODS AND MATERIALS}

This was a prospective cohort study in MICU at UTH, Lusaka, Zambia. Data was collected between August and November 2017 from patients 18 years and above and admitted for at least 24 hours in MICU for any medical or surgical condition. Patients admitted for less than 24 hours and re-admissions were excluded. Informed consent was obtained from the patient, next of kin or the Senior Medical Superintendent in case the patient is unknown or incapacitated. This clinical study was a consecutive case series with a random starting patient was picked who met the inclusion criteria then all eligible patients thereafter. A sample size of 100 was calculated based on the prevalence formulae $\mathrm{N}=\mathrm{Z} 2 \times \mathrm{P}(1-\mathrm{P}) /(\mathrm{E}) 2$ assuming a regional prevalence rate $(\mathrm{P})$ of $7 \%$, $\mathrm{Z}$ statistic $=1.96(95 \% \mathrm{CI})$ and confidence interval (E) of 0.05 .The patients were recruited in the order in which they were identified by meeting the inclusion criteria. The patients were enrolled upon admission to MICU and followed up until either their transfer to the ward or death within the Unit. The first panel of investigations was collected upon admission to MICU. This data was obtained from the routine baseline investigations obtained from every patient admitted to the Unit which included a full blood count, renal function tests and liver function tests. The second set of investigations was collected 24 hours post admission to MICU. This set of investigations are part of the daily routine investigations of patients in a well-equipped institution. The blood samples were obtained from a peripheral vein in EDTA bottles for full blood count and heparinized bottles for renal function tests. The samples were analysed from the UTH main laboratory. Serum electrolytes were analysed using the Beckman CoulterAU480 analyser serial: 2013102691machine manufactured in 2013, Mishima, Japan. Ethical approval was sought from the University of Zambia Research Ethics Committee and granted on 28th November 2016. Ethical clearance number: UNZABREC (REF. NO.006-10-16). In addition, the patient's comorbidities, fluid balance sheet, and medications were obtained and entered together with the panel of investigations. Together this data was entered into a data collection sheet. The data collected was then computed into an excel spread sheet. Data from excel spread sheet was imported into STATA special edition 13.0 for statistical analysis. The data was summarized and expressed as mean, median and standard deviation.

Comparisons between means were done with paired t-test and non-parametric data with the Wilcoxon signed rank test. Logistic regression analysis was used to investigate the relationship between dependent(Mortality and Prolonged ICU stay)and independent(Electrolyte abnormalities) variables. A p - value $<0.05$ was considered statistically significant. 


\section{RESULTS}

A total number of one hundred (100) patients were included in this study with a mean age of 36.8 (SD $=12.1$ ). The majority of patients were males 55 (55\%) and in the age group of 18 to 73 years. The females were $45(45 \%)$ and in the age group of 20 to 70 years. The average length of stay was $5.5 \pm 4.7$ days in MICU until death/transfer to other wards. Of the total admission, $52 \%$ were transferred to other wards whilst $48 \%$ died in the unit. The mean value of sodium level was $136.7(\mathrm{SD}=8.9) \mathrm{mmol} / \mathrm{L}$ and $139.0(\mathrm{SD}=11.6) \mathrm{mmol} / \mathrm{L}$, on admission and 24 hours post admission respectively. This difference in serum sodium level was statistically significant with a $P$-value $=0.005$. The mean value of potassium level was $4.2(\mathrm{SD}=1.1) \mathrm{mmol} / \mathrm{L}$ and $4.3(\mathrm{SD}=1.1)$, on admission and 24 hours post admission respectively. This difference was not statistically significant $(\mathrm{P}$-value $=0.57)$. However, there were significant changes in hypernatremia, hypokalaemia, and hyperkalaemia in the first 24 hours with $\mathrm{p}$-values less than 0.001 for all three abnormalities.

Logistic regression was done separately for mortality as an outcome and prolong ICU stay as an outcome. Hypernatremia was shown to be associated with an increased risk of death $(\mathrm{p}=$ 0.015) in the Unit with OR: 4.30 (95\% CI, 1.3 13.9). However, hypernatremia was not shown to be associated with prolonged stay $(\mathrm{P}=0.44)$ in MICU. Hyponatremia was neither shown to be statistically associated with mortality $(\mathrm{P}=0.21)$ nor prolonged Intensive Care Unit stay $(\mathrm{P}=0.56)$ at 24 hours post admission. Neither hypokalaemia $(\mathrm{p}=$ $0.29)$ nor hyperkalaemia $(p=0.07)$ were associated with mortality at 24 hours post admission and there was no association with duration of stay in the unit, hypokalaemia $(\mathrm{P}=0.09)$ and hyperkalaemia $(\mathrm{P}=$ $0.49)$.

\section{DISCUSSION}

In this study, the most prevalent electrolyte abnormality at the point of admission was hyponatremia (36\%) followed by hyperkalaemia (28\%), hypokalaemia (24\%) and the least was hypernatremia (13\%). 24 hours post admission in the intensive care unit, there was no significant changes in potassium levels. However, there were significant changes in sodium levels with the notable change in patients with hypernatremia. There was a significant increase in the number of patients with hypernatremia by almost twice the number at 24 hours from the number recorded at admission. This reflects the study by Polderman 2 that "hypernatremia is an indicator of quality of care in Intensive Care Unit". One explanation for this blood picture is possible dehydration resulting from the high incidence of patients not fed orally $(65 \%)$ in the first 24 hours of admission2. This was mostly in surgical patients whose treatment plan required them to be zero per oral. Other losses may be related to active nasogastric or oral gastric tubes3. Hypernatremia may also be due to increased losses from the kidneys due to renal impairment in critically ill patients coupled with reduced water intake4,5. In medical patients, the commonest reason for not feeding or giving fluids orally was an active nasogastric or orogastric tubes6. The unit doesn't have a clear policy or guideline at which volume of aspirate should lead to restrictions of oral feeds and fluids. This might result in patients being starved and fluid restricted unnecessarily. Fluid replacement is key in maintaining fluid balance5. In MICU, there are only two working infusion pumps against ten patients in the unit. This poses a challenge in replacing fluids and titrating fluids as prescribed in MICU resulting in the developing of hypernatremia. Rosner5 stated that hypernatremia in the Intensive Care Unit is often iatrogenic and due to inadequate free water replacement of ongoing water losses.

Normal saline was a commonly used crystalloid in MICU despite having a high concentration of sodium and a tendency of causing hyperchloremic acidosis6. This could be the cause of hypernatremia in certain patients with impaired homeostasis7. In this study hypernatremia was also shown to be associated with poor outcome (mortality) but not with prolonged ICU stay. The unadjusted OR: 3.99 (95\% CI, $1.2-6.3$ ) of mortality within 24 hours of admission with sodium level $>145 \mathrm{mmol} / \mathrm{L}$. Rosner5 demonstrated that "hypernatremia present on admission or developing in ICU is an independent risk factor for poor prognosis". This was earlier documented by Bagshaw8 that "hypernatremia in hospitalized patients is iatrogenic and may contribute to serious morbidity and increased risk of death". Another study showed that "the development of hypernatremia is associated 
with adverse outcomes for patients developing hypernatremia in the ICU and hypernatremia could potentially be used as an indicator of quality of care in the medical ICU"9.

There was a slight decrease in the patients with hyponatremia, but it remained the second most common electrolyte abnormality at 24 hours post admission. Dammon10 reported findings that "there is a high prevalence of dsynatraemias at ICU admission and that even mild to moderate abnormal concentrations are risk factors for ICU mortality". Despite being the most prevalent electrolyte abnormality, hyponatremia was not associated with mortality and prolonged ICU stay. This result is unlike the studies by Rosner5 and Bagshaw8 which showed an increased risk of mortality in patient with hyponatremia. This disparity can be due to the reduction in the number of patients with hyponatremia 24 hours post admission. The high frequency of hyponatremia can be attributed to administration of hypotonic fluids (5\% dextrose and $10 \%$ dextrose) and impaired water secretion and retention6.

The increase in incidence of hyperkalaemia after admission can be explained by the pre existing renal failure or indeed the developing renal failure with delay in dialysis5,7. Prevalence of hypokalaemia remained the same. Patients with hypokalaemia were postoperative patients for abdominal pathologies such as peritonitis and bowel obstruction9. Other patients with hyponatremia were on medical treatment with beta - blockers, insulin and adrenaline1. In this study hyponatremia, hypokalaemia and hyperkalaemia were all not shown to be associated with either poor outcome or prolonged duration of stay. This lack of statistical significance is probably a consequence of the weakness in the sample size of this study, or the degree of change in electrolyte in a 24-hour period may not have produced significant disturbances to support the already existing literature. Further research may need to be done with a larger sample size and to compare the changes at subsequent times e.g. 48 hours, 72 hours, or 5 days post admission.

As stated by Balcil fluid and electrolyte balance are one of the key issues in maintaining homeostasis in the body, and it also plays important roles in protecting cellular function". Therefore, a change in serum electrolytes in the various fluid compartments can result in impairment of cellular function and homeostasis. In the Intensive Care Unit, the patients are in a dynamic physiologic state which may rapidly deteriorate11. Hence, slight changes in the electrolyte and fluid status can have significant impact on the outcome and duration of stay in Intensive Care Unit if these patients are not appropriately and promptly managed 9 .

\section{CONCLUSIONS}

The results from this study shows that electrolyte and fluid disturbances can occur as early as 24 hours post admission into ICU. Therefore, electrolyte imbalances can occur as early as the first day of admission in ICU with fatal complications. Hyponatremia was the most prevalent abnormality in the patients admitted to the unit but there was a tendency of the blood picture to change towards hypernatremia as the patient stayed longer in the unit. There was a statistically significant change in serum sodium levels after 24 hours post admission but there was no statistically significant change in potassium level. Hypernatremia had a statistically significant association with mortality and therefore, correcting electrolyte imbalances in ICU patients is an urgent necessity and should not be delayed. We should therefore manage electrolytes better in ICU. Hence early treatment and correction of electrolyte disturbances can reduce the high mortality rate which currently stands at $48 \%$.

\section{DECLARATION OF INTEREST}

The authors report no conflicts of interest. The authors alone are responsible for the content and writing of this article.

\section{ACKNOWLEDGEMENT}

This study was made possible with the support of Professor Bellington Vwalika and Professor John Kinnear. Their unreserved availability, advice, insightful criticisms, guidance, understanding and patient encouragement aided the writing of this thesis in innumerable ways. I would also like to thank Dr Dylan Bould whose steadfast support of this thesis was greatly needed and deeply appreciated. 


\section{REFERENCES}

1. Balci AK, Koksal O, Kose A, Armagan E, Ozdemir F, Inal T, et al. General characteristics of patients with electrolyte imbalance admitted to emergency department. World $\mathrm{J}$ Emerg Me.2013; 4(2): 113 -116.

2. Poldeman KH, Schreuder WO, Strack van schijndel RJ, Thijs LG. Hypernatremia in the intensive care unit; an indicator of quality of care. Rev Med Interne. 1999; 27(8): 11051108.

3. Hessels L, Hoekstra M, Mijzen LJ, Vogelzang M, Dieperink W, Lansink AO, Et al. The relationship between serum potassium, potassium variability and in-hospital mortality in critically ill patients and a before-after analysis on the impact of computer-assisted potassium control. Crit Care 2015; 19(1). 4.

4. Sedlacek M, Schoolwerth AC, Remillard BD. Electrolyte disturbance in the Intensive Care Unit. Semin Dial. 2006;19(6):496-501.

5. Rosner $\mathrm{MH}$, Ronco C. Dysnatremias in Intensive Care Unit. ContribNephrol. 2010; 165: 292 - 298.

6. Perel P.Colloids versus crystalloids for fluid resuscitation in critically ill patients. Cochrane Database Syst Rev. 2013 Feb 28;(2):CD000567. doi: 10.1002/14651858. CD000567.pub6.
7. Sedlacek M, Schoolwerth AC, Remillard BD. Electrolyte disturbance in the Intensive Care Unit. Semin Dial. 2006;19(6):496-501.

8. Bagshaw SM, Townsend DR. Disorders of sodium and water balance in hospitalised patients. Can J Anaesth. 2009; 59(2): 151 159.

9. Lobo DN, Macafee DA, Allison SP. How perioperative fluid balance influences postoperative outcomes. Best Practice Res Clinical Anaesthesiology 2006; 20(3): 439 455.

10. Damon M, Diconne E, Souweine B, Ruckly S, Adrie C, Azoulay, et al. Prognostic consequences of borderline dsynatremia: pay attention to minimal serum sodium change. Crit Care. 2013; 17(1): R12

11. Kapoor d, Srivastava S, Singh P. Point of care blood gases with electrolyte and lactate in adult emergencies. Int $\mathrm{J}$ CritIllnInj Sci. 2014;4:216-222.

12. Stelfox HT, Ahmed SB, Khandwala F, Zygun $\mathrm{D}$, Shahpori R. The epidemiology of intensive care unit-acquired hyponatraemia and hypernatremia in medical-surgical intensive care. Crit care 2008; 12: R16

13. Lee, J. W. Fluid and Electrolyte Disturbances in Critically Ill Patients. Electrolyte and Blood Pressure Press. 2010; 8(2): 72 - 81. 


\section{TABLES AND FIGURES}

Chart 1: Flow chart of data management

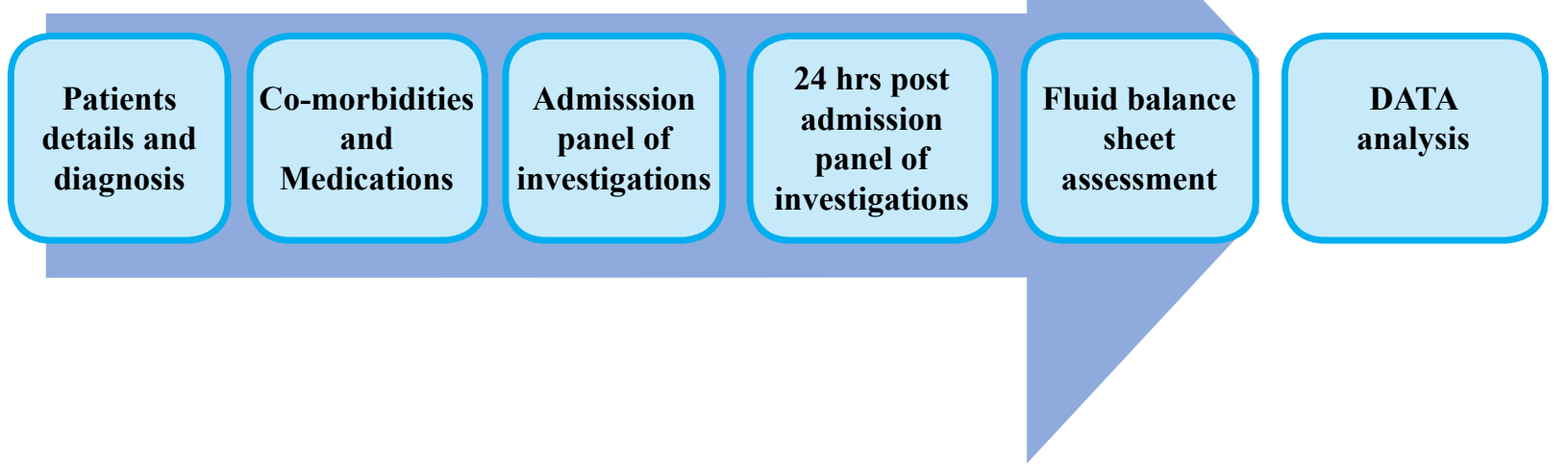

Table 1: Demographics characteristics

\begin{tabular}{|l|c|c|c|}
\hline \multirow{2}{*}{ Age (Years) } & Total & \multicolumn{2}{c|}{ Gender } \\
\cline { 2 - 4 } & & Male & Female \\
\hline Percentage (\%) & 100 & $55(55 \%)$ & $45(45 \%)$ \\
\hline Mean & 36.8 & 35.9 & 37.7 \\
\hline Median & & 34 & 35 \\
\hline Standard deviation & 12.1 & 11.9 & 12.1 \\
\hline
\end{tabular}

Table 2: Main Clinical variables

\begin{tabular}{|l|l|l|l|}
\hline & \multicolumn{1}{|c|}{ 0 hours } & 24 hours & P - value \\
\hline Serum sodium level & $136.7 \pm 8.9$ & $139.0 \pm 11.6$ & 0.005 \\
\hline Serum potassium level & $4.2 \pm 1.1$ & $4.3 \pm 1.1$ & 0.568 \\
\hline Serum sodium status & 0 hours & 24 hours & \\
\hline Hyponatremia & $128.6 \pm 5.4$ & $127.7 \pm 7.3$ & \\
\hline Normal & $138.5 \pm 2.8$ & $139.1 \pm 2.6$ & \\
\hline Hypernatremia & $142.3 \pm 7.2$ & $155.6 \pm 9.0$ & \\
\hline Serum potassium status & 0 hours & 24 hours & \\
\hline Hypokalemia & $3.1 \pm 0.4$ & $3.0 \pm 0.5$ & \\
\hline Normal & $3.9 \pm 0.3$ & $4.0 \pm 0.3$ & \\
\hline Hyperkalemia & $5.6 \pm 0 . .8$ & $5.5 \pm 0.9$ & \\
\hline
\end{tabular}


Table 3: Logistic regression results for increased risk of Mortality in MICU

\begin{tabular}{|l|c|c|c|}
\hline Independent variables & Odds Ratio & {$[95 \%$ Conf. Interval] } & $\mathbf{P}>|\mathbf{z}|$ \\
\hline Hyponatremia & 0.5 & $0.1-1.4$ & 0.210 \\
\hline Hypernatremia & 4.3 & $1.3-13.9$ & 0.015 \\
\hline \multicolumn{4}{|c|}{ Potassium } \\
\hline Hypokalaemia & 1.8 & $0.6-5.5$ & 0.294 \\
\hline Hyperkalemia & 2.5 & $0.9-6.7$ & 0.074 \\
\hline Constant & 0.5 & $0.2-1.1$ & 0.079 \\
\hline
\end{tabular}

Table 4: Logistic regression results for prolonged duration of Stay in MICU

\begin{tabular}{|l|c|c|c|}
\hline Independent variables & Odds Ratio & {$[95 \%$ Conf. Interval] } & $\mathbf{P}>|\mathbf{z}|$ \\
\hline \multicolumn{5}{|c|}{ Sodium } \\
\hline Hyponatremia & 0.8 & $0.3-2.0$ & 0.559 \\
\hline Hypernatremia & 1.6 & $0.5-4.8$ & 0.446 \\
\hline \multicolumn{5}{|c|}{ Potassium } \\
\hline Hypokalaemia & 2.7 & $0.9-8.3$ & 0.091 \\
\hline Hyperkalemia & 1.4 & $0.6-3.5$ & 0.485 \\
\hline Constant & 1.1 & $0.5-2.3$ & 0.799 \\
\hline
\end{tabular}

